\title{
AN INVERSE FINITE ELEMENT METHOD WITH AN APPLICATION TO EXTRUSION WITH SOLIDIFICATION
}

\author{
A. N. ALEXANDROU, N. R. ANTURKAR AND T. C. PAPANASTASIOU* \\ Department of Chemical Engineering, The University of Michigan, Ann Arbor, Michigan 48109, U.S.A.
}

\begin{abstract}
SUMMARY
The flow and solidification of planar jets are analysed by means of an efficient inverse isotherm finite element method. The method is based on a tessellation that is constructed by isotherms as characteristic co-ordinate lines transverse to the flow direction. Thus opposite sides of finite elements lie on isotherms. The method allows the simultaneous determination of the location of the isotherms with the primary unknowns, namely, the velocity, the pressure, the temperature and the location of the free surface. Thus the determination of the location of the solidification front (which is known to pose significant computational difficulties) is automatic. This facilitates the control of the location of the solidification front by controlling macroscopic variables such as the flow rate, the cooling rate and the capillary design. The location of the solidification may then be suitably chosen to influence the frozen-in orientation and structure in extrusion of highperformance materials such as composites and polymers, in continuous casting of metals and in growth of crystals.
\end{abstract}

KEY WORDS Inverse finite elements Solidification Extrusion Metal casting

\section{INTRODUCTION}

The extrusion of materials is an important process used extensively in polymer and composite material forming, in continuous casting of metals and glass and even in food processing. The molten material is forced through a capillary and allowed to expand freely and solidify by cooling. Because of their importance, extrusion problems have received and are still receiving a great deal of attention by many investigators. The process has been studied extensively in order to gain a deeper understanding of the physics and ultimately to develop the means for design, control and optimization. Yet control of industrial extruders and casters is largely based on intuition and experience.

In the past decades, with the increasing availability of large computers, computer-aided analysis became an important tool of design and optimization of many industrial processes. Computer-aided analysis requires a complete mathematical description of the process and an accurate numerical solution to the mathematical model. Real processes are, however, often very complex and their analysis poses considerable mathematical and computational challenges. One class of complex problems is free boundary problems in which, along with the traditional field variables such as the velocity, the pressure and the temperature, unknown geometric parameters need to be computed. Free boundary problems are highly non-linear with two types of nonlinearities; those due to unknown geometric parameters, such as the melt/solid interface in

* Author to whom correspondence should be addressed.

0271-2091/89/050541-15\$07.50

(C) 1989 by John Wiley \& Sons, Ltd.

Received 16 March 1988 Revised 28 July 1988 
solidification processes, and those due to the convective terms in the equations of motion and energy. Extrusion is a free boundary problem which includes all possible kinds of complexities: the location of the jet free surface and the location of the solidification front. Additional difficulties are the flow singularity at the exit of the die and the transition from the adherence of the melt to the die wall to perfect slip after solidification. In the case of polymer extrusion the situation is further complicated by the viscoelastic character of the liquid. The latter case is beyond the scope of this work.

A number of studies in the literature deal with non-isothermal extrusion of Newtonian ${ }^{1-4}$ and non-Newtonian ${ }^{5}$ liquids. None of these studies, however, accounts for melt solidification. The temperature dependence is introduced by augmenting the traditional Navier-Stokes equations with the energy equation and by treating the viscosity as a decreasing function of temperature.

The process of solidification itself, however, independently of extrusion, has been studied extensively. More recently, significant work has been done in relation to crystal growth. These processes are associated with the formation of single crystals which are the basis of manufacturing semiconductor devices. ${ }^{6-9}$ Several numerical methods have appeared in the literature dealing with the solidification of the materials and with the determination of the melt/solid interface. Most numerical techniques are for Stefan-like problems where the equations are linear with only the temperature as the unknown variable (e.g. Pearson ${ }^{10}$ ). The solution to the linear equations is obtained using a variety of numerical methods such as finite difference or finite element. The free boundary is obtained by successive iterations after each solution of the linear problem, until a condition associated with the solidification front is satisfied. The convergence of these methods is linear. ${ }^{11}$ A review of the various finite element techniques used to study Stefan-like problems has been presented by Lynch and Grey. ${ }^{12}$

In a detailed paper, Ettourney and Brown ${ }^{13}$ proposed four methods based on the finite element techniques to analyse solidification. All four methods were applied to a Stefan-like extrusion problem where the equations were made linear by dropping the non-linear convective terms. The problem was simplified further by neglecting both the die swell at the exit of the die and the change in the material properties across the solidification front. The first method, known as the isotherm method, was applied to a variable mesh and the equations were solved in the original domain without any co-ordinate transformation. The discontinuous energy flux boundary condition along the solidification front was relaxed and instead the isotherm condition was satisfied. Then, by successive approximations on the shape of the solidification front, the converged solution was obtained. When the interfacial energy flux condition along the melt solid interface was used as the distinguished condition, the method did not always converge. Furthermore, in the cases where it converged, the method had slower convergence than the rest of the methods examined in the same paper. By using a co-ordinate transformation which was a function of the position of the melt/solid interface, the solution to the solidification problem was obtained using three alternative methods: the kinematic isotherm method, the isotherm-Newton method and the energy flux method. In these methods, the choice of the distinguished condition was critical. In general, when the isotherm condition was satisfied, the results were more accurate than those which were obtained by balancing the energy fluxes along the solidification line. The most accurate results were obtained using the isotherm-Newton method, which in addition provided the fastest (quadratic) convergence. In the isotherm-Newton method the isotherm condition was satisfied at each node along the solidification line by equating the melt temperature at those nodes.

Two more finite element methods to solve the non-linear free boundary problem of crystal growth using a variable computational mesh have been proposed by Cuvelier and Driessen. ${ }^{7}$ In the first method one of the boundary conditions was relaxed by fixing the shape of the interface 
and then the non-linear problem was solved using the finite element method. The solution obtained was used to compute a new boundary that satisfied the relaxed boundary condition. The procedure was repeated until the iteration converged. This method is computationally expensive, because in addition to Newton iteration of the conservation equations it demands several Picard iterations to correct the free surface profile. The second method introduced the location of the free boundary as an additional degree of freedom and the solution to the complete system was obtained by using a quasi-Newton iteration with Broyden's updating technique. The method was applied to the analysis of heat transfer in a liquid in an open vessel under gravity. In this application there was only one phase present and the serious problem of coupling the melt with the solid was not dealt with.

A computationally efficient scheme based on Newton iteration and solidification parameterization was developed by Onoda ${ }^{14}$ to study continuous casting of metals. In casting of metals under gravity the solidification always occurs inside the die. In a related work in the area of metal casting, ${ }^{15}$ where the initial stress rate method is used to analyse the elasto-visco-plastic flow, the free surface is computed by a shooting technique.

The purpose of this work is three-fold:

1. To develop a computationally effective and fast algorithm which can be easily incorporated in a process control package: the algorithm is based on a global Newton iteration which computes the position of the solidification front and the temperatures simultaneously, including the surface temperatures which can be measured.

2. To develop a friendly algorithm by reducing the mathematical complexities and the bookkeeping as much as possible, which arise mainly due to the various types of elements-elements adjacent to the free surface, elements adjacent to the solidification front, melt elements and solid elements - and due to the boundary conditions. The algorithm is based on a novel inverse isotherm finite element method (IIFEM) which sets the temperatures and finds their locations. The known temperature of the solidification is set and thus the isotherm of the solidification and the relative position of the melt and the solid elements are known a priori. The isotherms, including the solidification front, are permitted to slide along the boundaries until they acquire a permanent position which satisfies the energy equation.

3. To test the hypothesis of a movable solidification front inside and outside the die and the sensitivity of its location to the external temperature.

The basic idea is to discritize the flow domain into finite elements with perpendicular (across the flow) sides on isotherms. The equations which define the isotherms are then solved simultaneously with the conservation equations, which permits the simultaneous evaluation of the position of the isotherms along with the primary unknowns-the velocity, the pressure and the temperature. The underlying idea behind one of the methods, the direct isotherm finite element method (IFEM), is similar to the one that led to the development of the streamlined finite element method by Papanastasiou et al. ${ }^{16}$ to analyse flows of viscoelastic liquids. The other method, the inverse isotherm finite element method (IIFEM), is a new development and can have applications elsewhere as well, when the calculation of characteristic lines or surfaces in flow or temperature fields is required, for one purpose or another. Some similarity exists between these methods and the isotherm migration method of Crank and Pahle ${ }^{17}$ which is based on finite differences and the inversion of the energy equation. The development of the IIFEM was carried out independently of the isotherm migration method, the existence of which was discovered a posteriori. ${ }^{18}$ The isotherm migration method was used by Talmon et al. ${ }^{19,20}$ to solve the moving boundary problems in one dimension by finite differences. 


\section{GOVERNING EQUATIONS AND BOUNDARY CONDITIONS}

The geometry of the flow is shown in Figure 1. A melt is forced through a slit while being cooled by the surroundings. At or near the exit, melt solidifies and produces a solid sheet. The hot sheet is cooled further by convection at ambient temperature. The two different regions of liquid and solid state are labelled as $A$ and $B$ respectively.

The governing equations of motion in the co-ordinate system shown in Figure 1 are

$$
\begin{aligned}
\rho \mathbf{u} \cdot \nabla \mathbf{u} & =-\nabla p+\nabla \cdot\left[\mu\left(\nabla \mathbf{u}+\nabla \mathbf{u}^{\mathbf{T}}\right)\right], \\
\nabla \cdot \mathbf{u} & =0,
\end{aligned}
$$

where $u$ is the velocity vector and $p$ the pressure. To account for thermal and shear effects, the viscosity $\mu$ can be made shear- and temperature-dependent; however, here it is assumed constant.

To obtain the non-isothermal problem, equations (1) and (2) are augmented by the energy equation

$$
\mathbf{u} \cdot \nabla T=\alpha \nabla^{2} T+(v / C) \Phi_{v},
$$

where $T$ is the temperature, $\alpha$ the thermal diffusivity, $v$ the kinematic viscosity and $C$ the heat capacity. The viscous dissipation function $\Phi_{v}$ is defined as

$$
\Phi_{v}=2\left[\left(\frac{\partial u}{\partial x}\right)^{2}+\left(\frac{\partial v}{\partial y}\right)^{2}\right]+\left(\frac{\partial u}{\partial y}+\frac{\partial v}{\partial x}\right)^{2} .
$$

Besides the four unknowns $u, v, T$ and $p$, the elevation of the free surface, $h(x)$, and the location of the solidification front, $S(y)$, are a priori unknowns. The kinematic condition that the free surface remains a material surface provides the additional equation needed to estimate the elevation of the free surface, i.e.

$$
\mathbf{n} \cdot \mathbf{u}=0
$$

where $\mathbf{n}$ is the unit normal vector.

The location of the solidification front, itself a characteristic line, is more difficult to determine because it depends on the solution of a highly non-linear system of equations with the additional complexity of a discontinuity in the thermal gradient across the solidification front of a priori unknown location. The solidification front is an isothermal line defined by the equation

$$
\mathbf{t} \cdot \nabla T=0,
$$

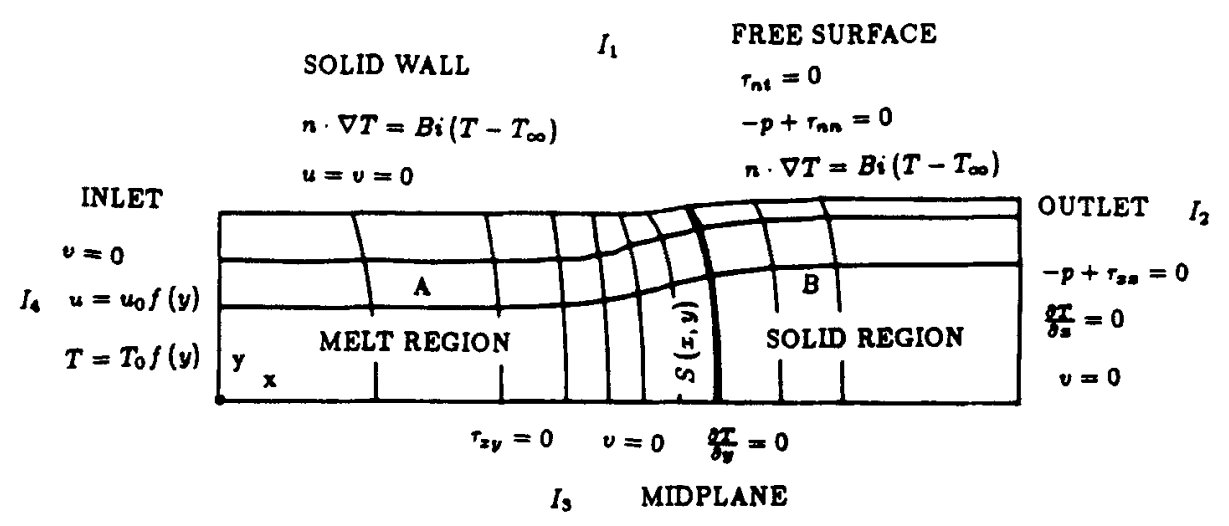

Figure 1. Flow domain and boundary conditions. The domains $A$ and $B$ represent the melt and the solid region respectively 
where $t$ is the tangential vector along the melt interface. Equation (6) states that there is no heat conduction along the isotherm of tangent $t$. If an additional unknown, the distance of the isotherm from the inlet, is introduced, the transverse boundaries of the elements can be forced onto isotherms by equation (6) solved along these boundaries. The solidification front is further characterized by

$$
L+\kappa_{\mathrm{s}} \frac{\partial T}{\partial n}-\kappa_{\mathrm{m}} \frac{\partial T}{\partial n}+\mathbf{n} \cdot\left[\rho_{\mathrm{m}} C_{\mathrm{m}} \mathbf{u}-\rho_{\mathrm{s}} C_{\mathrm{s}} U\right]=0
$$

where $L$ is the latent heat of solidification and $\kappa$ the conductivity. The subscripts $\mathrm{m}$ and $\mathrm{s}$ refer to the melt and solid states respectively. The discontinuity in the thermal gradients is an essential characteristic of the solidification process and must be accounted for in the analysis of flows with phase change. The latent heat $L$ accounts for the change in the density, the heat capacity and the velocity across the solidification front and is zero when there is no phase change. In the present work this discontinuity is included in the formulation and satisfied exactly along the solidification front as explained below.

Following the solidification, the material properties change and the effects due to viscosity vanish. In the solid region the extrudate moves as a solid body with constant axial velocity $U$, which is different from the velocity of the melt at the solidification front, the free surface levels off and heat transfer by conduction becomes the dominant effect. The solidified melt is then described by using the appropriate energy equation

$$
\rho_{\mathrm{s}} C_{\mathrm{s}} U \frac{\partial T}{\partial x}=\kappa_{\mathrm{s}}\left(\frac{\partial^{2} T}{\partial x^{2}}+\frac{\partial^{2} T}{\partial y^{2}}\right),
$$

which replaces equation (3). A discontinuity of the free surface profile is induced across the solidification front. The discontinuity is vanishingly small for small thermophysical changes across the solidification front as well as for solidification far downstream from the contact line, where the free surface becomes planar even before solidification. This discontinuity is therefore not detectable in the figures with the results.

The boundary conditions are shown in Figure 1. Due to symmetry, the analysis is performed on the upper half of the domain. The flow at the inlet is taken to be fully developed. Consequently, $u$ is parabolic and $v$ is zero. The temperature distribution at the inlet is obtained from the solution of the one-dimensional heat conduction along the $y$-direction. At the midplane, $v$ and the shear traction vanish. At the exit, $v$ and the axial traction are assumed to be zero. At the free surface, in the absence of surface tension, the traction is zero. The melt adheres to the solid wall (no-slip) whereas the solid sheet slips perfectly. The free surface and the wall are exposed to the surroundings and cool by convection. The formulation allows for variable heat transfer coefficient along these boundaries to account for different heat transfer rates along the solid wall and the free surface.

\section{THE ISOTHERM FINITE ELEMENT METHOD (IFEM)}

In the IFEM the location of the nodal points of the mesh is assumed to be unknown, except at the fixed intersections of the solid wall and the free surface with the isotherms. They are determined by solving the isotherm condition (equation (6)) along the transverse boundaries together with the rest of the equations in each element as shown in Figure 2. Therefore the transverse boundaries of the elements remain on the isotherms. The isotherm corresponding to the solidification tempe- 


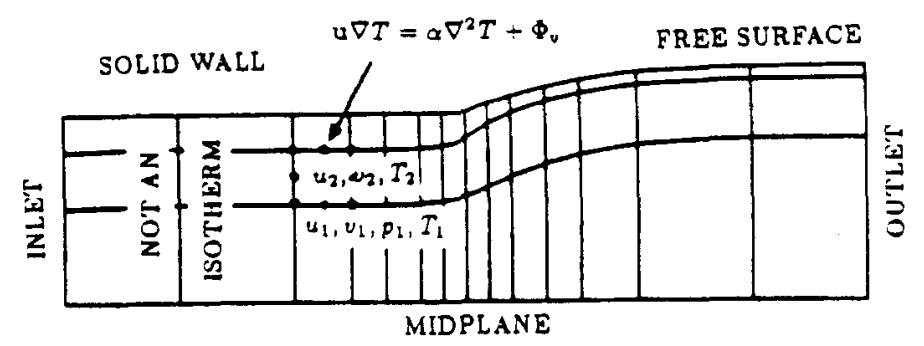

(a)



(b)

Figure 2. Finite element tessellations of (a) a conventional method and (b) of the IFEM. The additional equation $\mathrm{t} \cdot \nabla T=0$ is solved along the vertical sides of the element and determines the distance of the coinciding isotherm from the inlet

rature, $T_{0}$, defines the location of the solidification front and therefore the relative position of the elements in the melt and the solid region. This approach completely couples the melt and the solid regions. The solution is then obtained simultaneously by global Newton iteration for both regions without any trial-and-error numerical procedure to determine the location of the solidification front.

The velocity components $u$ and $v$ and the temperature $T$ are expanded in terms of biquadratic finite element basis functions $\phi_{i}$, and the pressure $p$ in terms of bilinear finite element basis functions $\psi_{i}$ as usual. The free surface and the isotherms are expanded in terms of quadratic finite element basis functions

$$
\begin{aligned}
& y=h(x)=\sum h_{i} \phi_{i}[\xi(x, y), \eta=1], \\
& x=S(y)=\sum s_{i} \phi_{i}[\xi=1, \eta(x, y)],
\end{aligned}
$$

where $h_{i}$ and $s_{i}$ are the unknown nodal distances of the free surface and the isotherms from the midplane and the inlet respectively.

When the divergence theorem is invoked and the boundary conditions of Figure 1 are applied, the following residuals result:

$$
R_{x}^{i}=\iint\left[-\rho\left(u \frac{\partial u}{\partial x}+v \frac{\partial u}{\partial y}\right) \phi_{i}+\left(-p+\mu\left(2 \frac{\partial u}{\partial x}\right)\right) \frac{\partial \phi_{i}}{\partial x}+\mu\left(\frac{\partial u}{\partial y}+\frac{\partial v}{\partial x}\right) \frac{\partial \phi_{i}}{\partial y}\right] \mathrm{d} x \mathrm{~d} y=0
$$




$$
\begin{aligned}
R_{y}^{i}=\iint\left[-\rho\left(u \frac{\partial v}{\partial x}+v \frac{\partial v}{\partial y}\right) \phi_{i}+\left(-p+\mu\left(2 \frac{\partial v}{\partial y}\right)\right) \frac{\partial \phi_{i}}{\partial y}+\mu\left(\frac{\partial u}{\partial y}+\frac{\partial v}{\partial x}\right) \frac{\partial \phi_{i}}{\partial x}\right] \mathrm{d} x \mathrm{~d} y=0 \\
R_{\mathrm{c}}^{i}=\iint\left(\frac{\partial u}{\partial x}+\frac{\partial v}{\partial y}\right) \psi_{i} \mathrm{~d} x \mathrm{~d} y=0 \\
R_{\mathrm{k}}^{i}=\int\left(v-u \frac{\partial h}{\partial y}\right) \phi_{i}(\xi, \eta=1) \mathrm{d} s=0, \\
R_{\mathrm{m}}^{i}=\int(\mathbf{t} \cdot \nabla T) \phi_{i}(\xi=1, \eta) \mathrm{d} s=0 \\
-\iint\left[\rho_{\mathrm{m}} C_{\mathrm{m}}\left(u \frac{\partial T}{\partial x}+v \frac{\partial T}{\partial y}\right) \phi_{i}-\mu\left[2\left(\frac{\partial u}{\partial x}\right)^{2}+2\left(\frac{\partial v}{\partial y}\right)^{2}+\left(\frac{\partial u}{\partial y}+\frac{\partial v}{\partial x}\right)^{2}\right] \phi_{i}\right. \\
\left.+\kappa_{\mathrm{m}}\left\{\frac{\partial T}{\partial x} \frac{\partial \phi_{i}}{\partial x}+\frac{\partial T}{\partial y} \frac{\partial \phi_{i}}{\partial y}\right\}\right] \mathrm{d} x \mathrm{~d} y-\kappa_{\mathrm{m}} \int \mathrm{n} \nabla \cdot T \phi_{i} \mathrm{~d} s=0, \\
R_{\mathrm{s}}^{i}=\iint \rho_{\mathrm{s}} C_{\mathrm{s}}\left(U \frac{\partial T}{\partial x}\right) \phi_{i}+\kappa_{\mathrm{s}}\left[\frac{\partial T}{\partial x} \frac{\partial \phi_{i}}{\partial x}+\frac{\partial T}{\partial y} \frac{\partial \phi_{i}}{\partial y}\right] \mathrm{d} x \mathrm{~d} y-\kappa_{\mathrm{s}} \int \mathbf{n} \nabla \cdot T \phi_{i} \mathrm{~d} s=0 .
\end{aligned}
$$

The boundary integral that appears in the energy equation needs to be evaluated at all boundaries. The integral $I_{1}$ on the upper boundary is equal to the energy flux from the solid wall and the free surface and so it is replaced by

$$
I_{1}=\kappa \int \mathrm{n} \nabla \cdot T \phi_{i} \mathrm{~d} s=\int h_{\mathrm{c}}\left(T^{*}-T\right) \phi_{i} \mathrm{~d} s,
$$

where $h_{\mathrm{c}}$ is an appropriate heat transfer coefficient and $T^{*}$ the ambient temperature.

When the divergence theorem is applied to the energy equation in the two domains $\mathrm{A}$ and $\mathrm{B}$, two boundary integrals appear along the solidification isotherm of each side,

$$
I_{\mathrm{A}}=\kappa_{\mathrm{m}} \int \mathrm{n}_{\mathrm{A}} \cdot(\nabla T)_{\mathrm{m}} \phi_{i} \mathrm{~d} s \quad \text { and } \quad I_{\mathrm{B}}=\kappa_{\mathrm{s}} \int \mathbf{n}_{\mathrm{B}} \cdot(\nabla T)_{\mathrm{s}} \phi_{i} \mathrm{~d} s,
$$

where $\mathbf{n}_{\mathrm{A}}$ and $\mathbf{n}_{\mathrm{B}}$ are the unit normal vectors to the melt-solid interface and $(\nabla T)_{\mathrm{m}}$ and $(\nabla T)_{\mathrm{s}}$ the corresponding gradients. The integrals are proportional to the flux across the solidification line. When the elements are assembled to form the final system of equations, these boundary integrals are replaced according to equation (7) along the solidification line. In the present formulation, of course, this can be done very easily. Since the boundaries of the elements lie on isotherms, the elements that border with the solid-melt front are known a priori. In addition, the elements in the melt and the solid region are also known a priori. Thus, unlike earlier methods where one of the boundary conditions had to be relaxed initially, both equations (6) and (7) are directly satisfied along the solidification front in the present formulation. Equations (11)-(17) with their boundary conditions are nothing more than a classical system of non-linear equations to be solved directly and simultaneously by Newton iteration.

The domain was tessellated into 30 nine-node quadrilateral elements which were found to be sufficient for a mesh-independent solution as shown in the subsequent figures. Small elements were required near the exit of the die because of the large gradients.

The evaluation of the integrals was done numerically by a nine-point Gaussian integration. The resulting system of non-linear algebraic equations was solved by full Newton iteration:

$$
\mathbf{q}^{(n+1)}=\mathbf{q}^{(n)}-\mathbf{J}^{-1} \mathbf{R}\left(\mathbf{q}^{(n)}\right),
$$



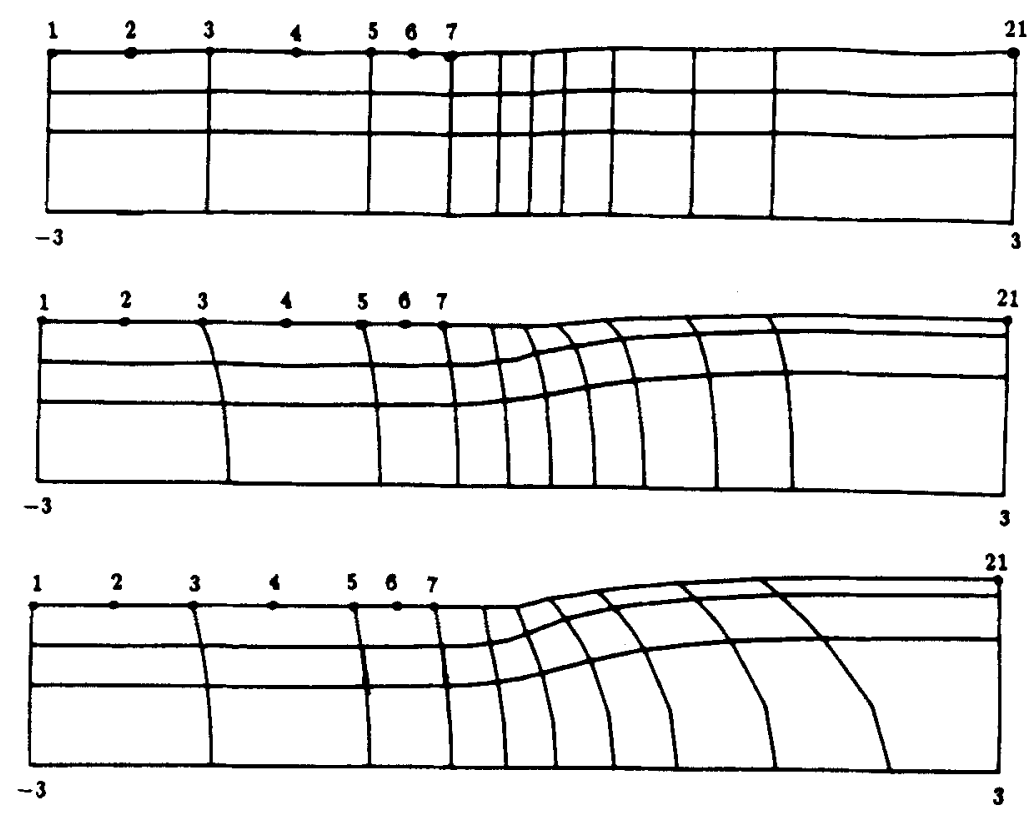

Figure 3. (a) The initial guess, (b) an intermediate step during the Newton iteration and (c) the converged solution of the IFEM in extrusion without solidification. The distances from the inlet to the nodes at the top boundary $1,2,3, \ldots, 21$ are fixed. The Prandtl number $P r=C \mu / \kappa$, is $0 \cdot 1$ and the Nusselt number, $N u=h_{\mathrm{c}} D / \kappa$, is $0 \cdot 1$

where $\mathbf{q}=\left[u_{1}, v_{1}, s_{1}, T_{1}, p_{1}, h_{1}, \ldots u_{N}, v_{N}, s_{N}, T_{N}, p_{N}, h_{N}\right]$ is the vector of the unknowns and $\mathbf{J}=\partial \mathbf{R} / \partial \mathbf{q}$ is the Jacobian of the residuals $\mathbf{R}$ with respect to the unknowns $\mathbf{q}$. The resulting banded system of linear algebraic equations at each Newton iteration step was solved by a frontal solver $^{21}$ on an APOLLO computer. It took approximately four minutes for each of the four or five Newton iterations.

The IFEM was first tested in non-isothermal extrusion without solidification. Figure 3 shows the initial guess for the mesh. The spacing of the isotherms is specified and kept constant throughout the Newton iteration along the solid wall and the free surface. Elsewhere, the isotherms rearrange themselves as the Newton iteration progresses and new free surface nodal elevations $h_{i}$ and new isotherm distances $s_{i}$ become available at each iteration. Figures 3(b) and 3 (c) show an intermediate tessellation and the final tessellation at which the Newton iteration converged quadratically to within four iterations with error in $\mathbf{R}$ less than $10^{-6}$.

The disadvantage of IFEM and other similar methods that appear in the literature is the necessity to fix the location of the isotherm along the wall and the free surface. For example, in the previous application the $x$ co-ordinate of point 5 was specified to be $-1 \cdot 0$. The value of its dimensionless temperature, with respect to the solidification temperature, obtained from the solution of the problem was 1.2426. Since the spacing of the isotherms of specified temperature along the wall and the free surface, and more importantly of the solidification front, is not known a priori, it is impossible to control the spacing of the isotherms on the wall and the free surface to correspond to certain temperatures, including the solidification temperature. Although this can be repaired by successive interpolation of the solidification front from the adjacent isotherms, the procedure is not computationally efficient.

The inverse isotherm finite element method (IIFEM) eliminates the above limitation completely, while preserving all the advantages of the IFEM. 


\section{THE INVERSE ISOTHERM FINITE ELEMENT METHOD (IIFEM)}

In the IFEM and in conventional finite element methods the question "what is the temperature (or the velocity or the pressure) at a specific location (the nodes of the elements)? is addressed. In contrast, the IIFEM addresses the question 'at what location (the nodes of the elements) does the temperature have a specific predefined value?'. This is the origin of the name of the method. Simplified versions of this approach were developed by Crank and Pahle ${ }^{17}$ and Crank and Gupta $^{22}$ and were used by Talmon et al. ${ }^{19,20}$ to solve one-dimensional static problems involving phase change by inverting the governing analytical equations. The system is much more complicated in this analysis and therefore not amenable to inversion. Instead, the energy equation, which is a function of position $\mathbf{r}$ and temperature $T$, is solved for $\mathbf{r}(T)$ instead of $T(\mathbf{r})$. In other words, the governing energy equation is equation (16) itself. Since the nodal temperatures are fixed, the remaining unknowns are the positions of the nodes. Thus equation (16) is solved for these unknowns by linearization with respect to these unknowns in order to form the Jacobian of the Newton iteration. The rest of the governing equations are treated as in the IFEM, the isotherm equation being dropped as discussed below.

Since the transverse boundaries of the elements are required to remain on isotherms, the temperature field can be specified a priori everywhere in the domain. Therefore the temperature is constant along the transverse boundaries of unknowns locations, which are permitted to change as the Newton iteration proceeds. In this way the isotherm condition, equation (6), is satisfied identically (actually, it is replaced by the conditions that specify the temperature everywhere) and is dropped from the analysis. The energy equation is then solved simultaneously with the rest of

Temperature (but not location) specified
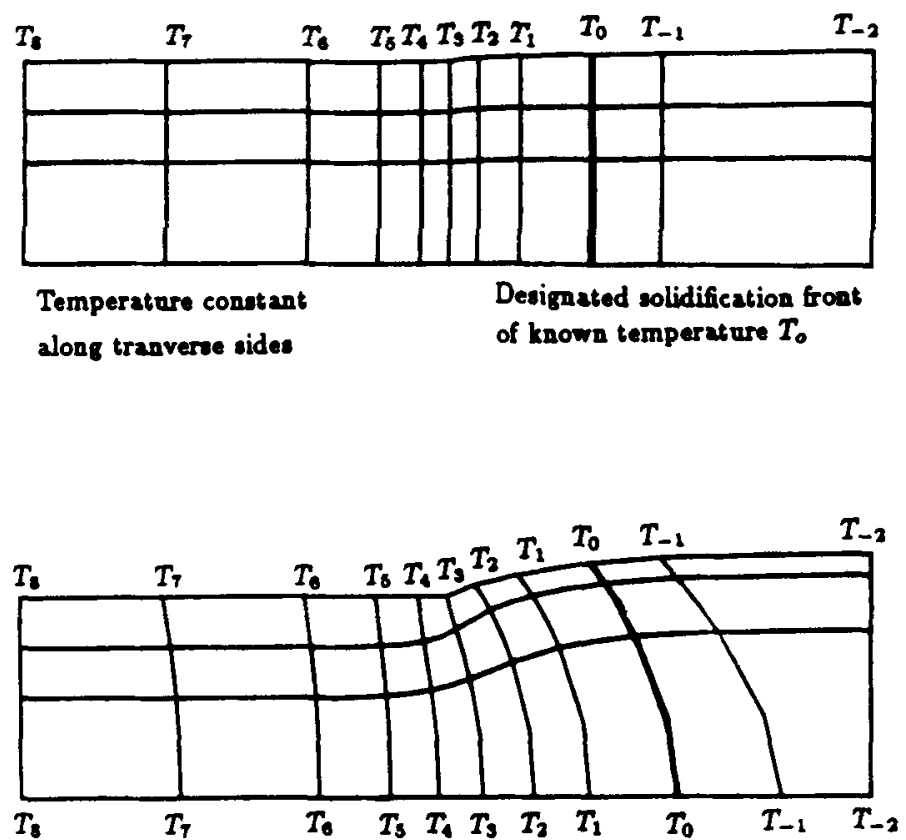

Figure 4. (a) The initial mesh guess and (b) the converged solution of the IIFEM. Constant temperature is specified along transverse sides, which are permitted to slide during the Newton iteration along the solid wall and the surface. One of the temperatures, $T_{0}$, corresponds to the solidification temperature 
the equations to obtain the positions of the isotherms of specified constant temperature. In other words, the $x$ co-ordinate of the nodal points becomes the dependent variable whereas the temperature is treated as the independent variable (Figure 4), thereby avoiding the inversion of matrices and subsequent computational cost.

The most important advantage of this formulation is the elimination of the requirement to specify the spacing of the isotherms along the solid wall and the free surface, which is replaced by the specification of the temperatures, including the a priori known temperature of solidification. The isotherms are allowed to slide along the solid wall and along the free surface in such a way that the predefined nodal temperatures remain constant along the sliding isotherm until the energy equation is satisfied upon convergence of the Newton iteration. The spacing of the isotherms can now be chosen to be as fine as one desires near the solidification front. By allowing the isotherm and the solidification front to change position freely, the algorithm can be easily used for process control.

Another advantage of the IIFEM is the reduction of the total number of unknowns due to the $a$ priori known temperatures of designated isotherms. The total degrees of freedom are 31 per element of melt region and 9 per element of solid region, as opposed to 37 and 15 respectively of the IFEM, and 31 and 9 respectively of conventional finite element methods plus the additional degrees of freedom of the solidification front. Thus the IIFEM contains less nodal unknowns than any other scheme and in addition it computes the isotherms, including the solidification front, at no extra cost.

To test the accuracy of the IIFEM, we reproduced the calculations obtained by the IFEM starting from two different initial guesses; the first one was the solution obtained by the IFEM as shown in Figure 3(c) and the second one is shown in Figure 5(a). In both cases the Newton iteration converged to the same solution, shown in Figure $5(\mathrm{~b})$, within two iterations in the former case and four iterations in the latter case with maximum nodal error less than $10^{-6}$.

Non-isothermal extrusion with solidification was examined next. Figure 6(a) shows the initial guess; the temperature profile is specified everywhere in the domain. The temperature of one isotherm corresponds to the solidification temperature (line SS). The governing equations of the melt state were applied to elements 1 to 12 whereas only the energy equation was applied to the
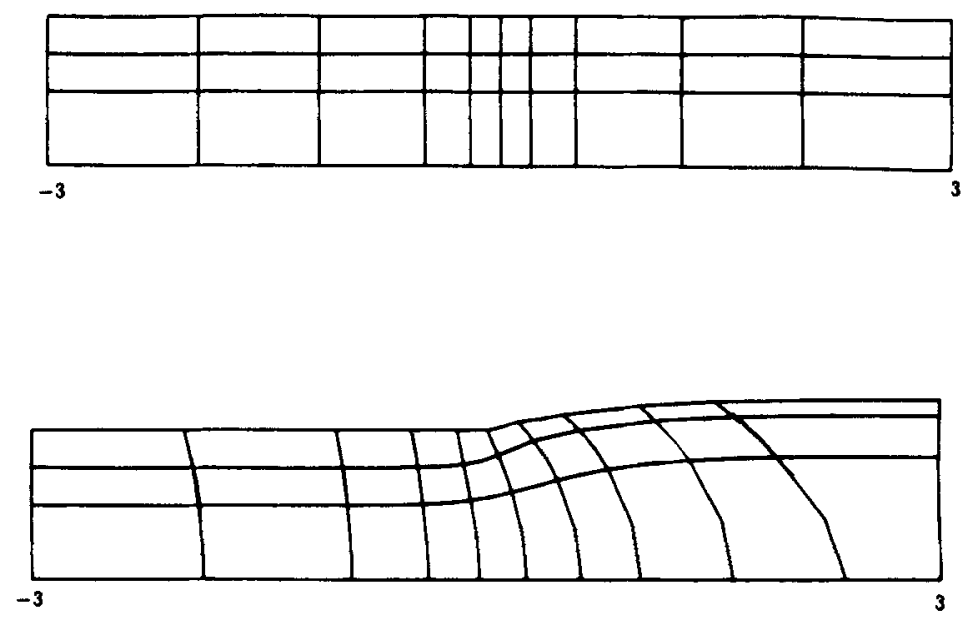

Figure 5. (a) The initial guess and (b) the converged solution of the IIFEM. The temperature profile obtained from the IFEM (Figure 3(c)) was used. The converged solution is identical to that of Figure 3(c) 

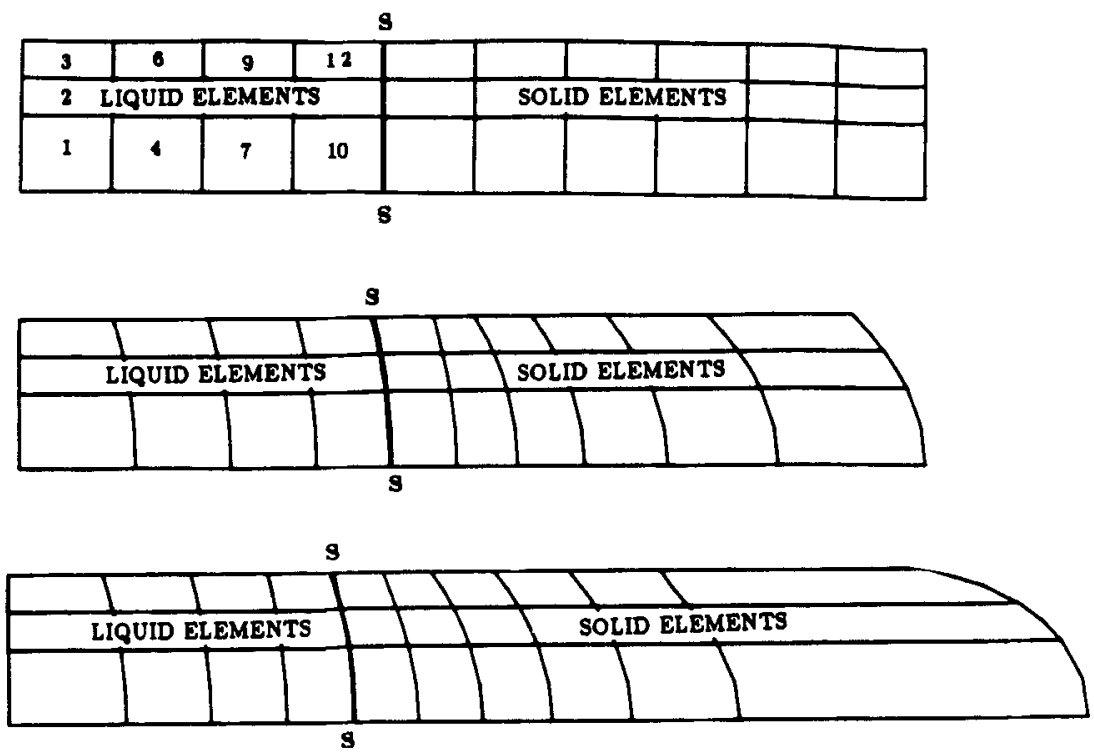

Figure 6. (a) The initial guess, (b) an intermediate step during the Newton iteration and (c) the converged solution of the IIFEM in extrusion with solidification. The parameters are $\operatorname{Pr}_{\mathrm{m}}=\operatorname{Pr}_{\mathrm{s}}=0.1, N u_{\mathrm{m}}=0.1$ and $N u_{\mathrm{s}}=0.5$

rest of the elements, in the solid region. The discontinuity condition of the thermal gradients (equation (8)) was applied along the solidification front SS. The rest of the boundary conditions were the same as in the IFEM. The Peclet number, $P e=C D U \rho / \kappa$, was $0 \cdot 1$ for both the liquid and the solid region. Figure 6(b) shows the tessellation after the first iteration. The mesh is automatically rearranged to conform to isotherms. The final mesh that is obtained with the converged solution is shown in Figure 6(c). Since the solidification occurs inside the die, there is no swelling of the extrudate.

The location of the solidification front depends on the operating conditions (flow rate, cooling rate), the die design and the material properties (viscosity, conductivity, diffusivity). Figure 7 shows the effect of the Prandtl number, $\operatorname{Pr}=C_{\mathrm{m}} \mu / \kappa_{\mathrm{m}}$, on the location of the solidification front. It is evident that different melts with different material properties will solidify at different locations even under the same flow conditions.

The solidification can be forced far downstream from the die exit, well into the extensional flow field (Figure 8). In this case molecular (or fibre) orientation parallel to the flow field can be achieved in the solid state. Production of solid parts with parallel orientation can have applications in a diversity of structural designs.

Other flow and processing situations that can be treated with the IIFEM may include:

1. Crystal growth. The application of the method to this process is obvious from the literature review discussed in the Introduction. In addition to the isotherms, the IIFEM can calculate isoconcentration lines of impurities or dopant materials.

2. Semiconductor modelling. The IIFEM can be used to determine the interfaces between Laplace and Poisson domains, for example, in field effect transistors. ${ }^{23,24}$ 


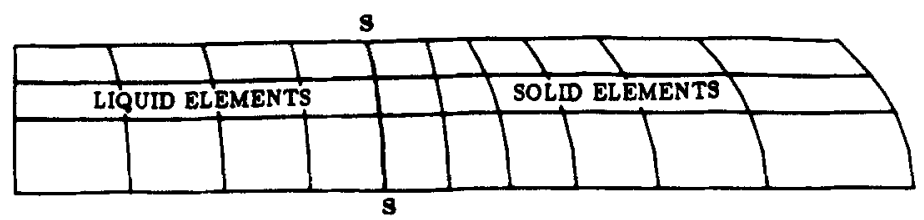

(a)

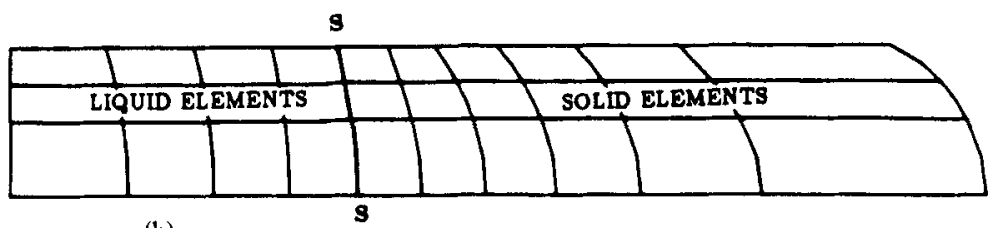

(b)

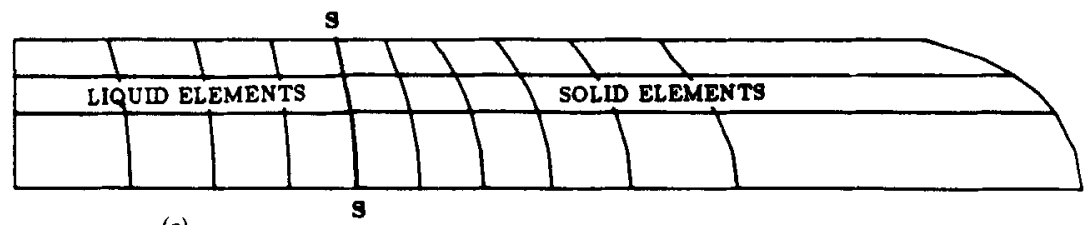

(c)

Figure 7. The IIFEM predictions for extrusion with solidification at various $\mathrm{Pr}$ and $\mathrm{Nu}$ numbers:

(a) $P r_{\mathrm{m}}=P r_{\mathrm{s}}=0.2, N u_{\mathrm{m}}=0.1, N u_{\mathrm{s}}=0.5$;

(b) $P r_{\mathrm{m}}=0.18, P r_{\mathrm{s}}=0.15, N u_{\mathrm{m}}=0.1, N u_{\mathrm{s}}=0.5$;

(c) $P r_{\mathrm{m}}=0.15, P r_{\mathrm{s}}=0.2, N u_{\mathrm{m}}=0.1, N u_{\mathrm{s}}=0.5$

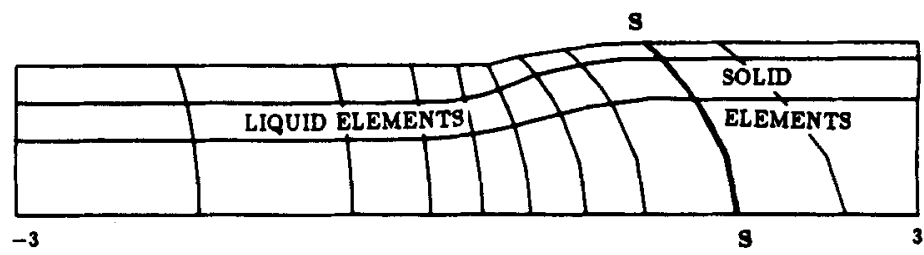

Figure 8. Under some operating conditions the melt may solidify outside the die for given material properties. Here $P r_{\mathrm{m}}=P r_{\mathrm{s}}=0.1, N u_{\mathrm{c}}=0.1, N u_{\mathrm{fs}}=0.5$ and $T_{\mathrm{s}}=0.45$

3. Continuous casting of metals and alloys. The application to this process is straightforward and the equations of motion are usually augmented by gravity terms. Convection and diffusion of impurities, gases and oxides may also be monitored and studied by means of isoconcentration lines. ${ }^{10,14,25}$

The weakness of the method is that it cannot be applied to flows with complicated closed isotherms, which are beyond the scope of this work. Isolated closed isotherms, however, can be easily handled by the IIFEM. Materials with no sharp melting point can be easily modelled by defining a solidification zone bounded by two isotherms corresponding to the lower and upper temperatures of the change of phase. 


\section{CONCLUSIONS}

Steady, non-isothermal extrusion of inelastic liquids, accompanied by solidification can be effectively analysed by means of a newly designed, inverse, isotherm, finite element method (IIFEM), that utilizes isotherms as tessellation co-ordinates. The method eliminates the necessity for tracking the solidification front by a trial-and-error procedure or else by complicated computations. The resulting non-linear system of equations, which satisfies all the appropriate boundary conditions, is solved once and for all by Newton iteration, which converges quadratically and determines all the unknowns, including the location of the solidification front, simultaneously.

The analysis showed that in principle it is possible to control the location of the solidification front by means of macroscopic process variables; locations of the solidification front were calculated from far upstream to far downstream distances from the die exit.

The generalization of the method to other situations is obvious. Examples include crystal growth to determine the isotherms, the isoconcentration lines and the solidification fronts; semiconductor operation to calculate isopotential lines and charge interfaces; casting, and others. The method as it stands cannot handle temperature fields dominated by complex patterns of closed isotherms.

\section{ACKNOWLEDGEMENTS}

This work was supported by a Rackham Faculty Award to T. C. Papanastasiou and by the Process and Reaction Engineering Program of the National Science Foundation, grant CBT8504607 .

\section{APPENDIX: NOTATION}

$\begin{array}{ll}C & \text { heat capacity } \\ D & \text { half-width of the channel } \\ h_{\mathrm{c}} & \text { heat transfer coefficient } \\ h(x) & \text { elevation of free surface from midplane } \\ I & \text { boundary integral in energy equation } \\ \mathbf{J} & \text { Jacobian of the residual } \mathbf{R} \text { with respect to unknown } \mathbf{q} \\ L & \text { latent heat of solidification } \\ \mathbf{n} & \text { unit normal vector } \\ N u & \text { Nusselt number }\left(h_{\mathrm{c}} D / \kappa\right) \\ p & \text { pressure } \\ P e & \text { Peclet number }(C D V \rho / \kappa) \\ P r & \text { Prandtl number }\left(C_{m} \mu / \kappa_{\mathrm{m}}\right) \\ \mathbf{q} & \text { vector of nodal unknowns } \\ \mathbf{R} & \text { vector of residuals } \\ R_{\mathrm{c}}^{i} & \text { continuity residuals } \\ \mathbf{R}_{\mathbf{i}}^{i} & \text { isotherm condition residuals } \\ R_{\mathrm{k}}^{i} & \text { kinematic residuals } \\ R_{\mathrm{m}}^{i} & \text { energy residuals in melt } \\ R_{\mathrm{s}}^{i} & \text { energy residuals in solid } \\ R_{x}^{i} & x \text {-momentum residuals } \\ R_{y}^{i} & y \text {-momentum residuals }\end{array}$




$\begin{array}{ll}s(y) & \text { distance of isotherm from inlet plane } \\ S(y) & \text { location of the solidification front } \\ \mathbf{t} & \text { unit tangent vector } \\ T & \text { temperature } \\ T_{0} & \text { solidification temperature } \\ T^{*} & \text { ambient temperature } \\ \mathbf{u} & \text { velocity vector } \\ u, v & x \text { and } y \text {-components of velocity } \\ U & \text { constant axial velocity } \\ x, y & \text { rectangular co-ordinates }\end{array}$

\title{
Greek symbols
}

$\begin{array}{ll}\alpha & \text { thermal diffusivity } \\ \kappa & \text { conductivity } \\ \mu & \text { viscosity } \\ \xi, \eta & \text { isoparametric co-ordinates } \\ \rho & \text { density } \\ \phi & \text { biquadratic basis function } \\ \Phi_{\mathrm{v}} & \text { viscous dissipation function, equation (4) } \\ \psi & \text { bilinear basis function }\end{array}$

\author{
Subscripts \\ $i \quad i$ th node \\ m melt state \\ s solid state
}

\section{REFERENCES}

1. K. R. Reddy and R. I. Tanner, 'On the swelling of extrudate plane sheets', J. Rheol., 22, 661 (1978).

2. H. B. Phuoc and R. I. Tanner, 'Thermally induced extrudate swell', J. Fluid Mech., 98, Part 2, 253 (1980).

3. E. Ben-Sabar and B. Caswell, 'Heat transfer effects in die-swell', J. Rheol, 25, 537 (1981).

4. B. P. Huynh, 'Some finite element studies of extrusion', J. Non-Newtonian Fluid Mech., 13, 1 (1983).

5. F. Sugeng, N. Phan-Thein and R. I. Tanner, 'A study of non-Newtonian extrudate swell by a mixed boundary element and finite element method', J. Rheol., 31, 37 (1987).

6. J. J. Derby and R. A. Brown, 'Finite element analysis of a thermal-capillary model for liquid encapsulated Czochralski growth', J. Electrochem. Soc. Solid State Sci. Technol. 132, 470 (1985).

7. C. Cuvelier and J. M. Driessen, 'Thermocapillary free boundaries in crystal growth', J. Fluid Mech., 169, 1 (1986).

8. W. E. Langlois, 'Convection in Czochralski growth melts', PCH: Phys.-Chem. Hydrodyn., 2, 245 (1981).

9. W. E. Langlois, 'A parameter sensitivity study for Czochralski bulk flow of silicon', J. Cryst. Growth, 56, 15 (1982).

10. J. R. Pearson, 'On the melting of solids near a hot moving interface with particular reference to beds of granular polymers', Int. J. Heat Mass Transfer, 19, 405 (1976).

11. J. M. Ortega and W. C. Rheinbolt, Iterative Solution of Nonlinear Equations in Several Variables, Academic Press, New York, 1970.

12. D. R. Lynch and W. G. Grey, 'Finite element simulation of flow in deforming regions', J. Comput. Phys., 36, 135 (1980).

13. H. M. Ettourney and R. A. Brown, 'Finite element methods for steady solidification problems', J. Comput. Phys. 49, 118 (1983).

14. M. Onoda (with L. E. Scriven), 'Continuous solidification of cast sheet with finite element method', M.S. Thesis, University of Minnesota, 1984.

15. P. R. Dawson and E. G. Thompson, 'Finite element analysis of steady-state elasto-visco-plastic flow by the initial stress-rate method', Int. j. numer. methods eng., 12, 47 (1978).

16. A. C. Papanastasiou, C. W. Macosko and L. E. Scriven, 'Streamlined finite elements and transit times', Finite Elements in Fluids, 6, 263 (1985).

17. J. Crank and R. D. Pahle, 'Melting ice by isotherm migration method', Bull. J. Inst. Math. Appl. 9, 12 (1973). 
18. L. E. Scriven, Personal communication, 1987.

19. Y. Talmon, H. T. Davis and L. E. Scriven. Progressive freezing of composites analyzed by isotherm migration methods', AICHE J., 27, 928 (1981).

20. Y. Talmon, H. T. Davis and L. E. Scriven, 'Moving boundary problems in simple shapes solved by isotherm migration', AICHE J., 29, 795 (1983).

21. P. Hood, 'Frontal solution program for unsymmetric matrices', Int. j. numer methods eng., 10, 379 (1976).

22. J. Crank and R. S. Gupta, 'Isotherm migration method in two-dimensions', Int. J. Heat Transfer, 18, 1101 (1975).

23. Y. Kagawa, T. Murai and $O$. Matsumoto, 'Finite element iterative techniques for determining the interface boundary between Laplace and Poisson domains', Int. j. numer. methods eng., 19, 315 (1983).

24. W. Fichtner, D. J. Rose and R. E. Bank, 'Semiconductor device simulation', SIAM J. Sci. Stat. Comput., 4, 391 (1983).

25. R. Sicgel, 'Control of solidification boundary in continuous casting by asymmetric cooling and mold offset', Int. $J$. Heat Mass Transfer, 28, 500 (1985). 